Abstract 01-S07.04 Table 1 Prevalences of CT and NG in swingers (M/ F) and MSM systematically screened on three anatomical sites

\begin{tabular}{|c|c|c|c|c|}
\hline & $\begin{array}{l}\text { Swinger total } \\
N=762 \% \text { (n) }\end{array}$ & $\begin{array}{l}\text { Swinger } M \\
N=386 \% \text { (n) }\end{array}$ & $\begin{array}{l}\text { Swinger } \mathbf{F} \\
\mathbf{N}=375 \% \text { (n) }\end{array}$ & $\begin{array}{l}\text { MSM } \\
N=597 \% \text { (n) }\end{array}$ \\
\hline Ст & $7.0 \%(53)$ & $7.0 \%(27)$ & $6.9 \%(26)$ & $9.9 \%(59)$ \\
\hline CT urogenital & $5.1 \%(39)$ & $5.2 \%(20)$ & $5.1 \%(19)$ & $2.3 \%(14)$ \\
\hline CT anorectal & $3.8 \%(29)$ & $2.1 \%(8)$ & $5.6 \%(21)$ & $8.2 \%(49)$ \\
\hline CT oropharyngeal & $0.8 \%(6)$ & $1.0 \%(4)$ & $0.5 \%(2)$ & $1.3 \%(8)$ \\
\hline GO & $2.6 \%(20)$ & $2.1 \%(8)$ & $3.2 \%(12)$ & $5.0 \%(30)$ \\
\hline GO urogenital & $0.9 \%(7)$ & $0.8 \%(3)$ & $1.1 \%(4)$ & $1.8 \%(11)$ \\
\hline GO anorectal & $0.5 \%(4)$ & $0.3 \%(1)$ & $0.8 \%(3)$ & $2.8 \%(17)$ \\
\hline G0 oropharyngeal & $1.6 \%(12)$ & $1.3 \%(5)$ & $1.9 \%(7)$ & $2.7 \%(16)$ \\
\hline
\end{tabular}

Conclusion MSM and female swingers have high prevalences of anorectal CT which are often diagnosed without a urogenital infection. Therefore these risk groups need a targeted screening strategies including anorectal testing. The prevalence of oropharyngeal STI is relatively low, but it is often an isolated infection and therefore missed by the current screening strategy.

\section{1-S07.05 RARE EVENT: RECTAL SPECIMEN COLLECTION FROM MALES FOR CHLAMYDIAL INFECTION IN THE USA}

doi:10.1136/sextrans-2011-050109.41

${ }^{1} \mathrm{G}$ Tao, ${ }^{1} \mathrm{~K}$ Hoover, ${ }^{2} \mathrm{~B}$ Body, ${ }^{2} \mathrm{M}$ Nye, ${ }^{1} \mathrm{C}$ Kent. ${ }^{1} \mathrm{CDC}$, Atlanta, USA; ${ }^{2}$ Laboratory Corporation of America, USA

Background US guidelines recommend annual Chlamydia screening of the rectum and urethra among men who have sex with men (MSM) with sexual exposure at these anatomic sites. Routine pharyngeal chlamydia screening is not recommended. About $6 \%$ of US males have a history of sex with men. The prevalence of rectal chlamydia has consistently been high among MSM with a history of receptive anal sex. One study indicated that up to $53 \%$ of chlamydial infections would be missed and not treated if only urethral testing was performed among MSM. We examined data from a large commercial laboratory corporation with a substantial share of the US market and testing available across all 50 states, that also offers rectal and pharyngeal testing with nucleic acid amplification tests (NAAT), to estimate the frequency and positivity of specimens collected from men by anatomic site for chlamydia testing.

Methods Data for all Chlamydia testing methods was obtained by a large commercial laboratory between June 2008 and July 2010. The data set was then queried to obtain testing performed on males and analysed for anatomic site, test type, test result and age. Urethral and urine specimens were assumed to be testing for urethral infections.

Results Chlamydia testing was performed on 227188 specimens from men: $98.2 \%$ urethral, $1.3 \%$ rectal, and $0.5 \%$ pharyngeal. The age distribution of those tested was: $2.4 \%<15$ years, $15.0 \% 15-19$ years, $15.7 \% 20-24$ years, $15.6 \% 25-29$ years, $12.5 \% 30-34$ years, $10.8 \%$ 35-39 years, $10.0 \% 40-44$ years, and $18.0 \%>44$ years. Chlamydia positivity varied by anatomic site $(8.7 \%$ rectal, $6.9 \%$ urethral, and $3.5 \%$ pharyngeal $)$, and by age $(2.5 \%<15$ years, $13.2 \% 15-19$ years, $12.5 \% \quad 20-24$ years, $7.7 \% \quad 25-29$ years, $5.3 \% \quad 30-34$ years, $3.9 \%$ $35-39$ years, $3.1 \%$ 40-44 years, and $1.7 \%>44$ years). Of 2918 rectal specimens, $17.8 \%$ were tested by culture and $75.5 \%$ by NAAT, compared to $93.5 \%$ of urethral specimens tested by NAAT.

Conclusions Given the sexual practices of men in the US, it appears that only a small proportion of MSM are appropriately screened for rectal Chlamydia. Lack of FDA clearance for these specimen sources may contribute to the small proportion of MSM being screened for rectal Chlamydia. Interventions to increase rectal chlamydial testing among MSM are needed. Because data from this corporation represents a large proportion of testing in the US, it could be used to monitor changes in chlamydia testing practice among men in the future.

\section{1-S07.06 LYMPHOGRANULOMA VENEREUM IN THE UK: IS THERE EVIDENCE FOR RECTAL TO RECTAL TRANSMISSION? RESULTS OF A MULTICENTER CASE CONTROL STUDY}

doi:10.1136/sextrans-2011-050109.42

${ }^{1} \mathrm{H}$ Ward, ${ }^{1} \mathrm{~N}$ Macdonald, ${ }^{1} \mathrm{M}$ Ronn, ${ }^{2} \mathrm{G}$ Dean, ${ }^{3} \mathrm{~S}$ Pallawela, ${ }^{3} \mathrm{~A}$ Sullivan, ${ }^{4} \mathrm{~J}$ White, ${ }^{5} \mathrm{~A}$ Smith, ${ }^{6} \mathrm{P}$ French, ${ }^{7} \mathrm{C}$ Ison. ${ }^{1}$ Imperial College London, London, UK; ${ }^{2}$ Brighton and Sussex University Hospital, UK; ${ }^{3}$ Chelsea and Westminster Hospital, UK; ${ }^{4}$ Guys and St Thomas's Trust, UK; ${ }^{5}$ Imperial College Healthcare NHS Trust, UK; ${ }^{6}$ Central and Northwest London Trust, UK; ${ }^{7}$ Health Protection Agency, UK

Background The outbreak of lymphogranuloma venereum (LGV) in men who have sex with men (MSM) in the UK is ongoing, with over 500 cases diagnosed in 2010 alone. Control efforts have been limited by a lack of understanding of the epidemiology, particularly transmission. The majority of cases are rectal with small numbers of urogenital or pharyngeal infections. No significant reservoir of asymptomatic or undiagnosed infection has been identified. The possibility of rectal to rectal transmission is suggested by studies showing an association of LGV with fisting, use of sex toys and enemas. We aim to identify risk factors to better understand transmission.

Methods A case control study of LGV in six UK clinics from 2009 to the end of 2010. Confirmed cases of LGV in MSM were compared with symptomatic and asymptomatic controls. Clinical and behavioural data were collected using a web-based computerassisted self-interview and linked to web-based clinical report forms (CRF). We used a two-stage process to construct multivariable logistic regression models in order to control for confounding and interaction between risk factors.

Results We have recruited 99 cases, 86 symptomatic and 88 asymptomatic controls. There were 94 rectal cases (including one who also had pharyngeal LGV), two urethral and one genital ulcer (two CRFs outstanding). In univariate analysis, LGV was significantly associated with many factors; the strongest associations were with HIV infection, specific anal sex practices (including unprotected receptive and insertive intercourse, rimming, use of toys, fisting, douching), meeting partners through the internet or in a backroom, and use of stimulant drugs. In final multivariable logistic regression models HIV-positivity, fisting, younger age at first clinic attendance and recent use of methamphetamine remained significant predictors of LGV when compared to asymptomatic controls. Compared to symptomatic controls, unprotected insertive anal intercourse was the only significant risk factor in the final model.

Conclusions Men reporting both insertive and receptive anal sex practices are at highest risk of LGV. Rectal to rectal transmission may be occurring with the organism being transferred via toys, fingers and penises; transient colonisation of the urethra may explain the low incidence of urethral LGV. Improved hygiene measures may have an important role in reducing transmission.

Epidemiology oral session 8: STIs and HIV in female sex workers

\section{1-S08.01 HIGH HIV PREVALENCE WITHIN A GENERALISED EPIDEMIC; CONDOM USE, VIOLENCE, AND SEXUALLY TRANSMITTED INFECTIONS AMONG FEMALE SEX WORKERS IN DAR ES SALAAM, TANZANIA}

doi:10.1136/sextrans-2011-050109.43

${ }^{1} \mathrm{~N}$ Makyao, ${ }^{2} \mathrm{~A}$ Kangolle, ${ }^{2} \mathrm{~A}$ Gilly, ${ }^{1} \mathrm{G}$ Somi, ${ }^{3} \mathrm{M}$ Kazaura, ${ }^{4} \mathrm{E}$ Kim, ${ }^{5} \mathrm{M}$ Kibona,

${ }^{3}$ S Kamazima. ${ }^{1}$ National AIDS Control Programme Dar es Salaam, Tanzania, United 
Rep. of Tanzania; ${ }^{2}$ Centers for Disease Control and Prevention, Tanzania, United Rep. of Tanzania; ${ }^{3}$ Muhimbili University of Health and Allied Sciences MUHAS, Tanzania, United Rep. of Tanzania; ${ }^{4}$ Centres for Disease Control and Prevention, Atlanta, USA; ${ }^{5}$ Centres for Disease Control and Prevention, Tanzania, United Rep. of Tanzania

Background In Tanzania, adult HIV prevalence is estimated at 5.8\% and $9.3 \%$ in Dar es Salaam, well above the level of a generalised epidemic. Nonetheless, HIV infection and a constellation of associated factors are disproportionately borne by marginalised groups within the country. Female sex workers (FSW) are one such group; however, little is known about them in Tanzania.

Method Respondent-driven sampling was used to recruit self-identified FSWs aged $15+$ years, living in Dar es Salaam, who reported exchanging sexual intercourse for money in the past month. FSWs were interviewed about their social circumstances, STI risk behaviours and tested for HIV, Hepatitis B, C and other STIs. Point estimates and 95\% CI were adjusted for social network size and recruitment patterns using RDSAT.

Results From April to August 2010, 537 FSWs were recruited. Their median age was 29 years (range 15-63). Two FSWs (0.6\% 95\% CI: 0\% to $1.7 \%$ ) were $<10$ years when first selling sex (median age 17 years). Condom use at last sex was $82.1 \%$ (95\% CI: $77.0 \%$ to $87.0 \%$ ), but consistent use varied by type of partner; always condoms use with steady partner was $29.9 \%$ (95\% CI: $22.8 \%$ to $37.6 \%)$ and $65.1 \%(95 \%$ CI: $58.4 \%$ to $71.4 \%$ ) with one time client. The major reason for FSWs not using condoms was partner objection (44.1\%; $95 \%$ CI: $32.6 \%$ to $56.2 \%)$. The prevalence of STIs and viral hepatitis varied: HIV $30.2 \%$ (95\% CI: $24.7 \%$ to $36.4 \%$ ); Hepatitis B $5.6 \%$ (95\% CI: 3.7\% to $8.3 \%$ ); Hepatitis C $1.4 \%$ (95\% CI: $0.3 \%$ to $3.1 \%$ ), syphilis $2.1 \%$ (95\% CI: $0.8 \%$ to $3.4 \%$ ); Gonorrhoea $10 \%$ (95\% CI: $6.9 \%$ to $14.4 \%$ ), and Chlamydia $6.9 \%$ (95\% CI: $3.2 \%$ to $11.9 \%$ ). Many (292; $51.7 \%, 95 \%$ CI: $46 \%$ to $58 \%$ ) FSWs had experienced physical violence in the past 12 months and $34.7 \%$ (95\% CI: $28.7 \%$ to $40.6 \%$ ) had been forced to have sex. Few FSWs were IDU themselves (1.4\%; 95\% CI: $0.3 \%$ to $2.9 \%$ ), but $5.1 \%$ (95\% CI: $2.3 \%$ to $7.7 \%$ ) suspected regular clients, and $8.9 \%$ (95\% CI: $7 \%$ to $12 \%$ ) suspected one-time clients were IDU. These women had significantly higher prevalence of HIV (46.8\%; $95 \%$ CI: $31.5 \%$ to $63.9 \%$ compared to $23.1 \%$ (95\% CI: $16.4 \%$ to $29.3 \%$ ) for FSWs who did not suspect any partner.

Conclusion FSWs of Dar es Salaam have many-fold higher prevalence of HIV than the general population, with variable levels of other STIs. Their health situation calls for multifaceted services and structural interventions beyond HIV education and condom promotion. Preventive, care and treatment and personal level empowerment strategies are desperately needed among FSWs in Dar es Salaam, Tanzania.

\section{1-S08.02 WHO ARE THE WOMEN AT RISK OF HIV INFECTION IN RURAL ZIMBABWE AND HOW MANY ARE THERE? INSIGHTS INTO THEIR CHARACTERISTICS, LOCATIONS, AND BEHAVIOURS}

doi:10.1136/sextrans-2011-050109.44

\begin{abstract}
${ }^{1} \mathrm{~J}$ Elmes, ${ }^{2} \mathrm{~K}$ Nhongo, ${ }^{1} \mathrm{~T}$ Hallett, ${ }^{1} \mathrm{P}$ White, ${ }^{2} \mathrm{R}$ Mutsindiri, ${ }^{1} \mathrm{G}$ Garnett, ${ }^{2} \mathrm{C}$ Nyamukapa, ${ }^{1} \mathrm{~S}$ Gregson. ${ }^{1}$ Imperial College London, London, UK; ${ }^{2}$ Biomedical Research and Training Institute, Zimbabwe
\end{abstract}

Background In Zimbabwe's generalised epidemic, the relative importance of different risk groups to ongoing HIV transmission is unclear. We estimated population sizes and HIV prevalence of women involved in different levels of sex work activity in two socioeconomic locations (rural areas and commercial centres) in east Zimbabwe

Methods The baseline survey for a cohort study of 650 women at high risk of HIV infection (WR) was conducted in March 2010, using snowball and location-based methods to recruit women with any form of sexual exchange motivated by material reward including cash-per-act and informal credit-based arrangements. We restricted our analysis to a subset of WR who report receiving money for sex, excluding non-monetary transactions. WR were assigned to high, medium or low activity levels based on, among other factors, the frequency they report receiving money for sex and numbers of partners. CIs are based on Agresti-Coull 95\% estimation and $p$ values are for $\chi^{2}$ estimation unless otherwise stated.

Results WR were more common in commercial centres than in rural areas $11.1 \%$ (9.9\% to $12.4 \%$ ) vs $5.0 \%$ (4.3\% to $5.8 \%$ ). The pattern of sex work activity differed between locations $(p=0.049)$ and mean number of sexual partners, over 2 weeks, in high, medium and low activity WR were 4.1, 1.9 and 1.3 respectively (ANOVA test for trend: $\mathrm{p}<0.001)$. High activity WR comprised $2.4 \%(1.9 \%$ to $3.1 \%)$ of women in commercial centres vs $0.55 \%(0.34 \%$ to $0.89 \%)$ in rural; medium level, $7.1 \%$ (6.2\% to $8.2 \%$ ) vs $3.9 \%$ (3.3\% to $4.7 \%$ ); and low level, $1.56 \%(1.13 \%$ to $2.13 \%)$ vs $0.50 \%(0.30 \%$ to $0.82 \%)$,Abstract O1-S08.02 figure 1. Overall, HIV prevalence in WR was higher in commercial centres than in rural areas: $46.5 \%$ (95\% CI: $38.9 \%$ to $54.3 \%$ ) vs $37.8 \%$ ( $28.4 \%$ to $48.1 \%$ ) ( $p>0.1$ ). Mean HIV prevalence among high level WR was consistent across socioeconomic strata: 50\% (range: $35.8 \%-64.2 \%$ ). Prevalence among medium level WR tended to be higher in business centres: $47.5 \%$ (38.1\% to $57.2 \%)$ vs $35.2 \%$ (25.1\% to $46.8 \%$ ) in rural areas. In contrast, HIV prevalence in low level WR was higher in rural areas: $44.4 \%$ (18.8\% to $73.4 \%)$ vs $36.4 \%$ (19.6\% to $57.1 \%)$.
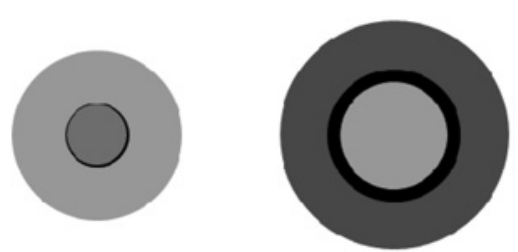

HIV prevalence (\%)

\section{Rural areas Commercial centres}

Abstract 01-S08.02 Figure 1 Relative size and HIV prevalence of the WR population in two socioeconomic locations in east Zimbabwe. Circle sizes are in proportion to the relative size of WR activity level; the inner ring represents low activity, the central ring high activity and the outer ring medium activity WR.

Conclusions Sex worker populations may be larger than previously measured in general population surveys in rural Zimbabwe. Given that HIV prevalence among WR is threefold higher than in the general population and the underestimated size of the sex worker population, sex work may be an increasingly important driver of HIV transmission in declining generalised rural African epidemics.

\section{1-S08.03 CONDOM USE DURING WORK TIME AMONG FEMALE SEX WORKERS IN BENIN}

doi:10.1136/sextrans-2011-050109.45

${ }^{1} \mathrm{~S}$ Diabaté, ${ }^{1} \mathrm{~A}$ Chamberland, ${ }^{2} \mathrm{~N}$ Geraldo, ${ }^{3} \mathrm{D} \mathrm{M}$ Zannou, ${ }^{4} \mathrm{M} \mathrm{M}$ Loembé, ${ }^{5} \mathrm{~S}$ Anagonou, ${ }^{6} \mathrm{~A}$ C Labbé, ${ }^{7} \mathrm{C}$ Tremblay, ${ }^{8} \mathrm{M}$ Alary. ${ }^{1}$ Centre de recherche, Centre hospitalier de I'Université de Montréal, Montréal, Canada; ${ }^{2}$ Dispensaire IST, Centre de santé de Cotonou I, Cotonou, Benin; ${ }^{3}$ Centre national hospitalier universitaire de Cotonou, Cotonou, Benin; ${ }^{4}$ nstitut de Médecine tropicale, Anvers, Belgium; ${ }^{5}$ Centre national hospitalier universitaire, Cotonou, Benin; ${ }^{6}$ Université de Montréal, Hôpital Maisonneuve-Rosemont, Montréal, Canada; ${ }^{7}$ Centre de recherche du Centre hospitalier de I'Université de Montréal, Montréal, Canada; ${ }^{8}$ URESP, Centre de recherche FRSO du CHA universitaire de Québec, Québec, Canada

Background Condom use remains the cornerstone of HIV prevention in sub-Saharan Africa where HIV transmission is 